\title{
INFLUÊNCIA DA LAVAGEM INTERMEDIÁRIA NA DESLIGNIFICAÇÃO COM OXIGÊNIO EM POLPA KRAFT
}

\section{INFLUENCE OF INTERMEDIATE WASHING IN OXYGEN DELIGNIFICATION ON KRAFT PULP}

\author{
Ivan Venson ${ }^{1}$ Alan Sulato de Andrade ${ }^{2}$ Umberto Klock ${ }^{1}$ Graciela Inés Bolzón de Muñiz \\ Silvana Nisgoski ${ }^{3}$ Gilson da Silva Cardoso ${ }^{4}$
}

\begin{abstract}
RESUMO
Na deslignificação com oxigênio, a lignina é removida da polpa através da aplicação de elevada pressão de oxigênio, em condições alcalinas, resultando em uma menor demanda por produtos químicos na etapa de branqueamento. O objetivo principal deste trabalho foi avaliar a influência do tipo de lavagem (convencional e ácida) entre dois estágios de deslignificação com oxigênio sobre a quantidade de lignina residual e o teor de metais de transição presentes em amostras de polpa kraft de pinus com número kappa inicial de 48,5. Observou-se a redução do número kappa e da viscosidade da polpa após o tratamento com $\mathrm{O}_{2}$ em duplo estágio, além do aumento da alvura final das polpas produzidas. O teor de metais da polpa durante a deslignificação com oxigênio sofreu influência do processo de lavagem, sendo que a lavagem ácida retirou maior quantidade de íons metálicos do que a lavagem convencional. Os melhores resultados foram obtidos com a retirada do elemento manganês. A lavagem ácida entre dois estágios de deslignificação contribuiu para o controle de metais de transição, o que otimizou a utilização de reagentes químicos do branqueamento nas etapas seguintes. Conclui-se que a lavagem intermediária melhorou o desempenho do oxigênio na deslignificação de polpas de número kappa elevado realizada em dois estágios.
\end{abstract}

Palavras-chave: lavagem da polpa; deslignificação com oxigênio; número kappa elevado; metais de transição.

\section{ABSTRACT}

In the oxygen delignification lignin is removed from the pulp by applying high pressure oxygen, under alkaline conditions, resulting in a lower demand for chemicals in bleaching. The main objective of this study was to evaluate the influence of washing (conventional and acid) between two stages of $\mathrm{O}_{2}$ on the amount of residual lignin and transition metal present in samples of Pine Kraft pulp with 48.5 initial kappa. The reduction of kappa number and pulp degradation after two stages of $\mathrm{O}_{2}$ treatment with increase of final brightness on sheet produced were observed. The metal content of pulp during oxygen delignification was influenced by washing process, and the acid wash removed more percentage of metallic ions than conventional wash. The best results were obtained with the removal of the element manganese. The acid wash between two stages of delignification contributed to the control of transition metals, which optimize the use of bleaching reagents in the following steps. We concluded that the intermediate washing improves

1 Engenheiro Florestal, Dr., Professor do Departamento de Engenharia e Tecnologia Florestal, Universidade Federal do Paraná, Av. Pref. Lothário Meissner, 900, CEP 80210-170, Curitiba (PR), Brasil. venson@ufpr.br/klockuer@gmail.com

2 Engenheiro Industrial Madeireiro, Dr., Professor do Departamento de Engenharia e Tecnologia Florestal, Universidade Federal do Paraná, Av. Pref. Lothário Meissner, 900, CEP 80210-170, Curitiba (PR), Brasil. alansulato@gmail.com

3 Engenheira Florestal, Dra , Professora do Departamento de Engenharia e Tecnologia Florestal, Universidade Federal do Paraná, Av. Pref. Lothário Meissner, 900, CEP 80210-170, Curitiba (PR), Brasil. gbmunize@ufpr.br/ nisgoski@ufpr.br

4 Engenheiro Químico, Msc., Doutorando do Programa de Pós-Graduação em Engenharia Florestal, Universidade Federal do Paraná, Av. Kennedy, 66, CEP 84261-400, Telêmaco Borba (PR), Brasil. gilson.cardoso@pr.senai.br

Recebido para publicação em 8/07/2011 e aceito em 22/04/2014

Ci. Fl., v. 25, n. 4, out.-dez., 2015 
the performance of the oxygen delignification of high kappa number pulps performed in two stages.

Keywords: pulp washing; oxygen delignification; high kappa; transition metals.

\section{INTRODUÇÃO}

A deslignificação com oxigênio consiste na reação da polpa com oxigênio e álcali $(\mathrm{NaOH})$, visando à remoção de fração da lignina remanescente após o cozimento e lavagem da polpa marrom. O oxigênio atua preferencialmente sobre as estruturas livres da lignina causando sua fragmentação, resultando em maior solubilidade e facilidade de remoção desse material (ALA-KAILA e REILAMA, 2001).

A primeira utilização industrial do oxigênio ocorreu na África do Sul em 1971 (DE SOUZA et al., 2002; BOUCHARD et al., 2003) e na década seguinte teve início o comércio desse reagente químico para aplicação em estágios de extração alcalina (REEVE, 1996).

A redução da demanda de cloro ativo, nos estágios subsequentes do branqueamento, e a redução da carga poluente do efluente, tendo em vista que a matéria orgânica dissolvida nesta etapa é recuperada e utilizada como fonte de energia, são as principais vantagens da utilização do oxigênio. No entanto, tem como desvantagens a elevação da carga de sólidos para o sistema de recuperação, além do alto custo de instalação e da limitada capacidade de deslignificação (pouco seletivo em relação ao cloro ou ao dióxido de cloro) (BERRY, 1996).

O branqueamento químico com oxigênio tem como limitante a despolimerização oxidativa da celulose. Além disso, a presença de íons metálicos na polpa, e na água usada no processo, provoca reações secundárias que precisam ser controladas, e ainda não são completamente compreendidas. Os radicais $\mathrm{OH}$ e $\mathrm{O}_{2}$ ou $\mathrm{OOH}$ são ativados durante as reações e o conhecimento da química desses radicais ainda está em estágio de desenvolvimento (REEVE, 1996; GIERER, 1997; SUESS, 2010).

O desempenho da deslignificação com oxigênio pode ser medido também pela quantidade de lignina lixiviável, pois o comportamento transitório da lignina residual, nas fibras de polpa kraft, consiste numa combinação da difusão natural e da lixiviação alcalina (ALA-KAILA e REILAMA, 2001).

Diferentes estudos convergem no aspecto de que a deslignificação para polpas de coníferas é limitada em torno de 40-50\%, devido à baixa seletividade do oxigênio e à dificuldade na transferência de massa eficiente entre uma fase gasosa e a fase sólida. Bassa et al. (2008) relatam que a taxa de deslignificação em polpas de coníferas são mais elevadas (45-55\%) em comparação com polpas de folhosas (30-35\%). As polpas de coníferas possuem maior teor de lignina e menor conteúdo de ácidos hexenurônicos, além de um maior número kappa inicial para a polpa que é alimentada na deslignificação com oxigênio. Assim, para aumentar a eficiência do processo tem-se usado duas sequências de oxigênio, o que permite um aumento na taxa de deslignificação.

Para polpas de folhosas a deslignificação com oxigênio fica limitada entre 25 e $35 \%$ pois estas normalmente são produzidas em números kappa baixos. Além disso, no caso das folhosas, parte do número kappa é atribuída à presença de ácidos hexenurônicos e outras duplas ligações (resinas, celulose oxidada), enquanto que o número kappa das coníferas é quase em sua totalidade composto de lignina, susceptível ao ataque do oxigênio (SUESS, 2010).

A eficiência do estágio com oxigênio pode ser melhorada pela adequada otimização das variáveis operacionais do processo, que incluem tempo, temperatura, carga de álcali, carga de oxigênio, consistência, pressão de reação, entre outros (ARGYROPOULOS e LIU, 2000). A taxa de deslignificação é diretamente proporcional ao aumento da carga de álcali, ocorrendo de forma linear até $2 \%$ de $\mathrm{NaOH}$ e a eficiência de deslignificação cresce, linearmente, com o aumento da temperatura (ZOU et al., 2000).

As causas de limitações que ocorrem durante as reações de deslignificação por oxigênio vêm sendo investigadas, e na procura pelas razões da inatividade da lignina, o enfoque são as estruturas aromáticas condensadas e não condensadas. Muitas dessas estruturas (difenilmetano, fenilcoumaril, 5-5 bifenila) foram determinadas como razoavelmente resistentes nas condições de deslignificação com oxigênio. As não condensadas parecem favorecer a deslignificação, enquanto que as condensadas tendem a ser mais resistentes (GELLERSTEDT e LINDFORS, 1987; ARGYROPOULOS e LIU 
2000; MOE e RAGAUSKAS 1999; CHEN e LUCIA, 2003).

Além das variáveis operacionais e da inatividade da lignina, o tipo e concentração de íons metálicos presentes na madeira e na polpa, também contribuem para um comportamento diferenciado durante o processo de branqueamento, especialmente quando são utilizados reagentes derivados do oxigênio. Os metais também podem ser adsorvidos pela polpa, através da água de processo, dos reagentes e do contato com os equipamentos utilizados na sua produção. Os íons metálicos podem estar presentes na polpa, associados aos ácidos hexenurônicos, através de forças coulômbicas e químicas, principalmente, devido à presença da dupla ligação no anel desses ácidos, o que favorece a sua capacidade quelante. A remoção desses grupos ácidos reduz a afinidade da polpa por íons metálicos, permitindo a redução do consumo de reagentes e da quantidade de agentes quelantes usados (BRASILEIRO et al., 2001).

Um dos fatores que mais afetam a deslignificação com oxigênio é o tipo e a quantidade de metais de transição presentes na polpa. Durante a fase de redução do oxigênio para água é gerado peróxido de hidrogênio, que é decomposto cataliticamente pelos íons dos metais de transição (MCDONOUGH, 1996; BOUCHARD et al., 2011).

A lavagem da polpa é uma operação importante entre as etapas de branqueamento (POTUCEK e PULCER, 2006) e tem como principal objetivo remover materiais orgânicos e inorgânicos dissolvidos que irão atrapalhar o estágio subsequente e aumentar o consumo dos reagentes. Quando a lavagem da polpa não é eficiente ocorre arraste de licor negro até o estágio de oxigênio e que afeta o resultado na deslignificação (SUESS, 2010). Como as condições ótimas de químicos variam nas sucessivas fases, um propósito muito importante da lavagem é deixar as condições mais favoráveis incluindo a modificação do $\mathrm{pH}$, o conteúdo de metais da polpa e a temperatura (SILLANPÄÄ, 2005). Uma boa lavagem pode ser combinada com reduzido consumo de água e químicos, reduzindo a geração de efluentes e, indiretamente, o custo de sistemas de tratamento externos dos resíduos (SAIBABA et al., 2009).

Diferentes análises sobre o impacto do $\mathrm{pH}$ do filtrado e processo de lavagem concluíram que poucos estudos avaliam o efeito da acidificação na remoção da lignina (SKOTNICOVÁ e POTƯÈEK, 2002) e que esses têm influência na sorção e desorção dos elementos cálcio e magnésio na polpa (GEMZICKÁ et al., 2010).

Estudos indicam que uma lavagem eficiente da polpa é importante antes da determinação do número kappa, para obter um bom desempenho do processo de deslignificação alcalina com oxigênio (ALA-KAILA e REILAMA, 2001). Uma das maneiras de melhorar a eficiência da deslignificação com $\mathrm{O}_{2}$ seria mediante uso de uma lavagem ácida. A lavagem ácida é uma forma simples para remover metais de transição no branqueamento e posterior eliminação destes resíduos. Como desvantagem dessa lavagem há eliminação do magnésio, que tem efeito benéfico nos estágios com oxigênio e peróxido (FREDDO et al., 1999).

A remoção de metais de transição através de quelação ou estágio ácido antes da deslignificação com oxigênio é bem conhecida (BOUCHARD et al., 2011). Porém, poucos estudos foram conduzidos para verificar a ação da lavagem ácida intermediária entre dois estágios.

Estudos prévios de deslignificação com oxigênio têm sido realizados com polpas de baixo número kappa, em torno de 30 para coníferas e 20 para folhosas. Para polpas com kappa elevado, enfatizam-se os efeitos da temperatura, pressão do oxigênio e carga de álcali na taxa de deslignificação, mas poucos estudos foram realizados considerando o efeito do kappa inicial. Assim, este estudo teve como objetivo principal avaliar a influência do tipo de lavagem (convencional e ácida) entre dois estágios de deslignificação com oxigênio sobre a quantidade de lignina residual e teor de metais de transição presentes nas amostras de polpa kraft de pinus com número kappa inicial de 48,5.

\section{MATERIAL E MÉTODOS}

\section{Caracterização das amostras}

O estudo foi desenvolvido com polpas kraft não branqueadas de Pinus spp. produzidas na empresa Irani Celulose, em Santa Catarina. As amostras de polpas foram coletadas após as etapas de cozimento, lavagem e depuração. As principais características das amostras de polpas não branqueadas estão apresentadas na Tabela 1 .

A polpa não branqueada foi submetida ao processo de deslignificação com oxigênio em dois estágios em laboratório. As condições dos estágios são apresentadas na Tabela 2 .

As polpas, após serem submetidas ao 
TABELA 1: Características da polpa kraft.

TABLE 1: Kraft pulp characteristics.

\begin{tabular}{cc}
\hline Número Kappa & 48,5 \\
Viscosidade (mPa.s) & 52,0 \\
Alvura inicial (\% ISO) & 22,6 \\
\hline Teor de metais (mg/kg - ppm): & \\
\hline $\mathrm{Fe}$ & 64,3 \\
$\mathrm{Cu}$ & 3,5 \\
$\mathrm{Mn}$ & 86,8 \\
$\mathrm{Mg}$ & 280,0 \\
\hline
\end{tabular}

primeiro estágio com oxigênio, foram avaliadas sobre três condições de tratamento: $1^{\circ}$ ) Sem lavagem - SL; $2^{\circ}$ ) Com lavagem convencional - LC (com água destilada) e; $3^{\circ}$ ) Com lavagem ácida LA. Lucia et al. (2002) ao aplicarem uma sequência dupla de deslignificação em polpa de coníferas de kappa elevado $(56,2)$, avaliaram cargas de $\mathrm{NaOH}$ de $2.5,4,1$ e $5,8 \%$.

As polpas que não sofreram lavagem foram centrifugadas a uma consistência de 30\% e em seguida submetidas ao segundo estágio de deslignificação. A lavagem da polpa entre dois estágios de deslignificação com oxigênio foi realizada de duas maneiras: somente com água destilada (lavagem convencional) e água destilada com adição de ácido sulfúrico $\left(\mathrm{H}_{2} \mathrm{SO}_{4}\right), \mathrm{pH}=3$ (lavagem ácida). Na lavagem convencional, a polpa foi lavada abundantemente com $28 \mathrm{~m}^{3}$ de água destilada por tonelada de polpa seca. Na lavagem ácida, a consistência da polpa foi ajustada para 3\% e essa foi então tratada com ácido sulfúrico $0,1 \mathrm{~N}$, até alcançar $\mathrm{pH}=3$. Em seguida, as polpas foram transferidas para sacolas de polietileno, misturadas e acondicionadas em um banho termostático, onde permaneceram sob agitação por 30 minutos a $50^{\circ} \mathrm{C}$, e então lavadas com excesso de água destilada ( 28 $\mathrm{m}^{3}$ de água/ton de polpa a.s.).

\section{Parâmetros de controle avaliados}

$\mathrm{O}$ efeito da lavagem intermediária foi avaliado através dos seguintes parâmetros: número kappa, viscosidade e teor de metais da polpa. Também foi avaliada a alvura das folhas formadas. Foram determinadas as quantidades dos seguintes íons metálicos presentes nas polpas: Ferro $(\mathrm{Fe})$, Cobre $(\mathrm{Cu})$, Manganês $(\mathrm{Mn})$ e Magnésio $(\mathrm{Mg})$. A preparação das polpas para análise quantitativa de metais foi realizada através do método de digestão das cinzas em ácido clorídrico e a leitura em um espectrofotômetro de absorção atômica da marca Shimidzu modelo AA 6200. As metodologias empregadas estão listadas na Tabela 3.

\section{Análise estatística}

Para avaliar o grau de associação entre as variáveis, foram estimados e testados os coeficientes de correlação. Os resultados foram calculados para níveis de $5 \%$ de significância (95\% de confiança). Para a realização das análises estatísticas foi utilizado o software Statigraphics Centurium XV.

\section{RESULTADOS E DISCUSSÃO}

\section{Efeito da lavagem intermediária entre dois estágios de $\mathrm{O}_{2}$}

A Tabela 4 apresenta os resultados da influência da lavagem entre dois estágios de deslignificação com oxigênio no número kappa, viscosidade e na alvura das polpas estudadas. Ressalta-se, que os valores dos parâmetros avaliados

TABELA 2: Condições utilizadas nos estágios de deslignificação com oxigênio.

TABLE 2: Conditions applied on oxygen delignification stage.

\begin{tabular}{lcc}
\hline \multicolumn{1}{c}{ Condições } & Primeiro estágio $\mathrm{O}_{2}$ & ${\text { Segundo estágio } \mathrm{O}_{2}}$ \\
\hline Consistência (\%) & 10 & 10 \\
Tempo (min) & 60 & 45 \\
Pressão $\left(\mathrm{kgf} / \mathrm{cm}^{2}\right)$ & 4 & 4 \\
$\mathrm{MgSO}_{4} \cdot 7 \mathrm{H}_{2} \mathrm{O}(\%)^{1}$ & 0,1 & 0,1 \\
Carga de álcali $(\%)^{1}$ & $2,4,6$ & $2(\mathrm{SL}, \mathrm{LC}), 4(\mathrm{LA})$ \\
Temperatura reator $\left({ }^{\circ} \mathrm{C}\right)$ & $90,100,115$ & 100 \\
\hline
\end{tabular}

Em que: ${ }^{1}$ Em relação ao peso base seca da polpa; SL - Sem lavagem; LC - Lavagem convencional; LA - Lavagem ácida. 
TABELA 3: Procedimentos analíticos utilizados nos ensaios.

TABLE 3: Analytical procedures applied on tests.

\begin{tabular}{|c|c|}
\hline Parâmetro analisado & Metodologia empregada \\
\hline N. Kappa & TAPPI T236 om-99 \\
\hline Viscosidade da polpa & TAPPI T230 om-99 \\
\hline Alvura & $\begin{array}{l}\text { TAPPI T571 om-03 } \\
\text { TAPPI T519 om-02 } \\
\text { TAPPI T452 om-02 } \\
\text { TAPPI T525 om-02 }\end{array}$ \\
\hline Determinação de metais na polpa espectroscopia de absorção atômica & TAPPI T266 om-02 \\
\hline Cinzas da polpa para determinação de metais na polpa & TAPPI T211 om-02 \\
\hline
\end{tabular}

foram determinados após a conclusão do segundo estágio de deslignificação.

Estatisticamente não houve diferença no número kappa entre as polpas, mas avaliando numericamente observa-se uma pequena diferença entre a polpa que não sofreu lavagem (19) e aquela que sofreu lavagem convencional $(18,8)$. Porém, quando se compara com a lavagem ácida $(18,1)$ constata-se uma redução de quase um ponto $(0,9)$ no número kappa, o que resulta em uma queda de $4,7 \%$ deste parâmetro. Isso demonstra que uma lavagem eficiente da polpa pode acarretar ganhos neste parâmetro, através da retirada de fragmentos de lignina dissolvidos no licor e ainda presentes na polpa. Ressalta-se que a redução de um ponto do número kappa impacta na redução de custos com reagentes, energia e meio ambiente.

Em relação à alvura observa-se que ambas as lavagens (convencional e ácida) apresentaram aumento de alvura em $0,8 \%$ ISO em relação à polpa não lavada (33,4 \% ISO).

A lavagem ácida apresentou o menor número kappa, acompanhada de menor valor de viscosidade, e alvura igual à da lavagem convencional. Isso indica que a lavagem ácida melhorou a seletividade na etapa de deslignificação com oxigênio.

Comparando a lavagem ácida com a convencional, observa-se que a lavagem apenas com água destilada obteve a manutenção da viscosidade da polpa (22,1 mPa.s) enquanto que a ácida apresentou pequena redução desta propriedade (19,2 mPa.s).

Em relação à redução do número kappa, a lavagem ácida obteve melhor resultado, obtendo um valor levemente inferior que a lavagem convencional no controle destes parâmetros.

\section{Efeito da lavagem convencional nos metais de transição}

Os metais de transição que estavam presentes na madeira ainda permanecem na polpa após a polpação, e afetam fortemente o grau de deslignificação. Na madeira é normal que ocorra diferenças significativas no conteúdo de metais de transição. A taxa de metais depende da

TABELA 4: Efeito da lavagem entre os estágios de $\mathrm{O}_{2}$.

TABLE 4: Washing effect between $\mathrm{O}_{2}$ stages.

\begin{tabular}{lccc}
\hline \multirow{2}{*}{\multicolumn{1}{c}{ Parâmetros }} & \multicolumn{3}{c}{ Tratamento } \\
\cline { 2 - 4 } & Sem lavagem & Lavagem convencional & Lavagem ácida \\
\hline Número kappa & $19,0 \mathrm{a}$ & $18,8 \mathrm{a}$ & $18,1 \mathrm{a}$ \\
Viscosidade (mPa.s) & $22,3 \mathrm{a}$ & $22,1 \mathrm{a}$ & $19,2 \mathrm{a}$ \\
Alvura (\%ISO) & $32,6 \mathrm{a}$ & $33,4 \mathrm{a}$ & $33,4 \mathrm{a}$ \\
\hline
\end{tabular}

Em que: Médias seguidas pela mesma letra na linha não apresentam diferença estatística ao nível de $95 \%$ de probabilidade 
disponibilidade de traços de metais no solo, sendo muitos essenciais para o metabolismo das árvores (SUESS, 2010).

A Tabela 5 apresenta os resultados obtidos para o teor de metais de transição entre os estágios de oxigênio com lavagem convencional.

A polpa lavada de maneira convencional apresentou redução nas quantidades dos elementos manganês, cobre e magnésio, por outro lado, a presença de ferro aumentou.

A quantidade de metais de transição diminuiu com a aplicação da lavagem intermediária entre o primeiro e o segundo estágio. Os teores de ferro, cobre, manganês e magnésio foram reduzidos, e os mesmos extraídos da polpa junto aos fragmentos de lignina, carboidratos e extrativos. Esses foram retirados pela ação da lavagem, que carregou grande parte dos elementos presentes na polpa. O que demonstra que a lavagem intermediária contribuiu no controle de metais.

Considera-se que o aumento em termos absolutos nos teores do elemento ferro, presente na polpa após a deslignificação, é atribuído à contaminação gerada pelo próprio reator, no qual foi realizada a reação. Outra fonte de contaminação a ser levada em conta provém da água utilizada no ajuste de consistência, na diluição dos elementos adicionados à polpa e na lavagem da mesma.

Observou-se uma redução de manganês em 31,8\% após a lavagem, o que é benéfico para a polpa, tanto para a deslignificação por oxigênio quanto para utilização de peróxido de hidrogênio como agente oxidante.

O teor de magnésio aumentou em relação aos valores iniciais, após o primeiro estágio de $\mathrm{O}_{2}$, devido à adição de $0,1 \%$ de $\mathrm{MgSO}_{4} \cdot 7 \mathrm{H}_{2} \mathrm{O}$. Já a lavagem intermediária provocou a redução de $10,09 \%$ desse metal, mesmo assim o valor foi maior que o teor de magnésio inicial da polpa (280 mg/kg). Destaca-se que esta redução é indesejável, pois o elemento magnésio promove a proteção dos carboidratos e um teor baixo deste elemento contribui para a redução da viscosidade da polpa.

Yokoyama et al. (1999) em seus estudos, observaram que o ferro estimula a degradação do carboidrato utilizado em seus estudos, e que o efeito protetor, pela adição do magnésio, depende da relação entre os níveis de $\mathrm{Fe}$ e $\mathrm{Mg}$. Quando a relação $\mathrm{Mg} / \mathrm{Fe}$ foi 2, maior quantidade de carboidratos foi degradada, comparativamente à situação em que o magnésio não estava presente. Porém, quando a relação $\mathrm{Mg} / \mathrm{Fe}$ foi 16 , o carboidrato se degradou rapidamente. Neste estudo a relação $\mathrm{Mg} / \mathrm{Fe}$ no primeiro estágio foi de 7,3, e passou para 9,3 após a lavagem convencional e 5,4 após lavagem ácida.

Bouchard et al. (2011) demonstraram que a seletividade do oxigênio é influenciada principalmente pela concentração de Fe in situ mais do que os íons adicionados durante a deslignificação, enquanto que a concentração in situ de cálcio afeta a eficiência da proteção pelo magnésio.

A contaminação da polpa, principalmente pelo ferro ao longo do processamento, pelos equipamentos e pela água utilizada também foi relatada em outros trabalhos. Torres et al. (2005), ao investigarem o branqueamento da polpa de Pinus tecunumanii, encontraram valores de $76,9 \mathrm{mg} / \mathrm{kg}$ para $\mathrm{Fe}$ e $23,76 \mathrm{mg} / \mathrm{kg}$ para $\mathrm{Cu}$. Segundo os autores, os valores eram devidos ao suprimento de água, corrosão do digestor ou a grande quantidade destes metais no solo da região no qual as árvores foram plantadas. Já Salomão (2001) relatou a quantidade de metais em polpas kraft produzidas com mistura de cavacos de Pinus elliottii e Pinus taeda, que continham $23 \mathrm{mg} / \mathrm{kg}$ de $\mathrm{Fe}$ e $3,3 \mathrm{mg} / \mathrm{kg}$ de $\mathrm{Cu}$. O efeito negativo do ferro na viscosidade da polpa, durante a deslignificação por oxigênio, foi observado por Rabelo (2006) e atribuído à decomposição de peróxidos pelos íons de $\mathrm{Fe}_{2}+\mathrm{e} \mathrm{Fe}_{3}+$.

TABELA 5: Teor de metais entre os estágios de oxigênio com lavagem convencional.

TABLE 5: Metal content between oxygen stages with conventional washing.

\begin{tabular}{lcccc}
\hline \multirow{2}{*}{ Sequência das etapas } & \multicolumn{4}{c}{ Elementos $(\mathrm{mg} / \mathrm{kg}-\mathrm{ppm})$} \\
\cline { 2 - 5 } & $\mathrm{Fe}$ & $\mathrm{Mn}$ & $\mathrm{Cu}$ & $\mathrm{Mg}$ \\
\hline Polpa não branqueada & 64,30 & 86,80 & 3,50 & 280,00 \\
Após 1 $^{\circ}$ estágio O & 66,70 & 68,70 & 5,16 & 488,07 \\
Após lavagem convencional $^{\text {Após 2 }}{ }^{\circ}$ estágio $\mathrm{O}_{2}$ & 46,93 & 59,22 & 1,99 & 438,79 \\
\hline
\end{tabular}




\section{Efeito da lavagem ácida na retirada de metais de transição}

A Tabela 6 apresenta os teores dos metais de transição presentes na polpa após os tratamentos de deslignificação com $\mathrm{O}_{2}$ e submetidos à lavagem ácida.

A eficiência do tratamento ácido foi maior no controle de manganês, o qual diminuiu em $75,13 \%$ enquanto que, na lavagem convencional, a redução de $\mathrm{Mn}$ foi de $13,8 \%$. A redução de ferro foi de $13,7 \%$ na lavagem ácida e o mesmo se elevou após o primeiro estágio de $\mathrm{O}_{2}$, mostrando que provavelmente a polpa foi contaminada com este elemento durante a reação. Cabe mencionar que o elemento ferro apresenta maior afinidade de ligação com as fibras, sendo assim mais difícil retirá-lo somente com os sistemas de lavagem.

Por outro lado, o teor de cobre diminuiu após a lavagem ácida apresentando uma queda de $63,17 \%$. Este efeito é desejável, porque à medida que se reduz a presença de cobre, diminuem-se as possibilidades de degradação da polpa celulósica durante a deslignificação com oxigênio. Esse metal catalisa a decomposição do peróxido de hidrogênio intermediário formado durante a redução do oxigênio. Isto entra em consonância com Bouchard et al. (2011) que em outras pesquisas ressaltam que a adição de $\mathrm{Cu}^{\mathrm{II}}$ na polpa induz a uma forte degradação da celulose.

O elemento magnésio apresentou uma redução de $36,72 \%$ após a lavagem ácida, enquanto que a queda com a lavagem convencional foi de $10,09 \%$. Esta perda do elemento magnésio é considerada um efeito indesejado, pois ele atua como protetor da polpa celulósica e melhora a seletividade das reações. Bouchard et al. (2011) suportam a teoria de que o $\mathrm{Mg}$ protege os carboidratos durante a deslignificação com oxigênio através da formação de complexos Mg-carboidratos, que é favorecida por uma baixa concentração de ferro residual promovendo o mecanismo de encapsulamento.

\section{Comparação entre os tipos de lavagem}

Os resultados evidenciam que a lavagem contribuiu para retirar parte dos íons metálicos presentes, quando comparada com a polpa sem lavagem. Porém, as polpas mesmo sendo submetidas aos dois estágios de $\mathrm{O}_{2}$, permaneceram com níveis elevados de metais. A Tabela 7 apresenta os resultados dos teores de metais das polpas, em diferentes condições de lavagem.

As polpas que foram duplamente deslignificadas e não passaram pelo processo de lavagem, mantiveram níveis elevados de ferro e manganês, quando comparadas com as polpas lavadas, nas quais a quantidade dos dois elementos foi reduzida. A lavagem ácida apresentou melhor controle do íon manganês do que a lavagem convencional. Conforme ressaltado anteriormente, o elemento ferro apresenta maior afinidade de ligação com as fibras, sendo mais difícil retirá-lo somente com os sistemas convencionais de lavagem. Há que se lembrar de que houve contaminação por parte do reator, o que dificulta o estabelecimento de um balanço preciso do elemento ferro.

\section{CONCLUSÕES}

A deslignificação com oxigênio em polpas de número kappa elevado permite retirar grande quantidade de lignina residual presente. A lavagem da polpa após a aplicação de oxigênio contribui para retirar traços de lignina e outros contaminantes presentes no licor residual. Sendo assim, uma lavagem eficiente prepara a polpa para o segundo estágio de $\mathrm{O}_{2}$ direcionando a ação oxidante do

TABELA 6: Teores dos metais entre etapas de lavagem ácida e duplo estágio de $\mathrm{O}_{2}$.

TABLE 6: Metals between acid washing steps and double $\mathrm{O}_{2}$ stage.

\begin{tabular}{lcccc}
\hline \multirow{2}{*}{ Sequência das etapas } & \multicolumn{4}{c}{ Elementos $(\mathrm{mg} / \mathrm{kg}-\mathrm{ppm})$} \\
\cline { 2 - 5 } & 64,30 & 86,80 & 3,50 & $\mathrm{Mg}$ \\
\hline Polpa não branqueada & 66,70 & 68,70 & 5,16 & 480,00 \\
Após 1 $^{\circ}$ estágio de $\mathrm{O}_{2}$ & 57,52 & 17,08 & 1,90 & 309,84 \\
Após lavagem ácida $^{\text {Após 2 }}{\text { estágio de } \mathrm{O}_{2}}^{2}$ & 68,05 & 21,8 & 1,60 & 149,6 \\
\hline
\end{tabular}


TABELA 7: Teores de metais nas diferentes condições de lavagem intermediária.

TABLE 7: Metal contents in various intermediate washing conditions.

\begin{tabular}{lrccc}
\hline \multirow{2}{*}{ Tratamento } & \multicolumn{4}{c}{ Elementos $(\mathrm{mg} / \mathrm{kg}-\mathrm{ppm})$} \\
\cline { 2 - 5 } & $\mathrm{Fe}$ & $\mathrm{Mn}$ & $\mathrm{Cu}$ & $\mathrm{Mg}$ \\
\hline Sem lavagem & 78,1 & 68,9 & 3,8 & 242,2 \\
Lavagem convencional (água) & 46,9 & 59,2 & 1,9 & 439,7 \\
Lavagem ácida & 57,5 & 17,1 & 1,9 & 309,8 \\
\hline
\end{tabular}

oxigênio para a lignina ainda não atacada durante o primeiro estágio.

A polpa da madeira de pinus apresentou níveis elevados de metais de transição, principalmente ferro e manganês, o que comprova a necessidade de uma lavagem eficiente quando for submetida a processos de deslignificação ou branqueamento.

Devido ao fato de que a polpa de pinus apresentou níveis elevados de metais de transição, notadamente ferro e manganês, ficou demonstrado que a lavagem da polpa contribui para a remoção do manganês, sem a necessidade de aplicação de quelantes.

Conclui-se que a lavagem ácida entre os dois estágios foi eficiente no controle do manganês presente na polpa, sendo superior à lavagem convencional. Mostra-se que a aplicação de uma lavagem intermediária prepara a polpa para o $2^{\circ}$ estágio de $\mathrm{O}_{2}$ potencializando seu efeito oxidante, uma vez que a polpa terá reduzido seus teores de fragmentos de lignina residual e de metais. Além disso, a retirada de íons de metais de transição previne reações secundárias que podem consumir o oxigênio. Por outro lado, a lavagem ácida apresenta o efeito indesejado de necessitar uma maior carga de álcali no segundo estágio de deslignificação para obter o pH alcalino para a reação.

\section{REFERÊNCIAS BIBLIOGRÁFICAS}

ALA-KAILA, K., REILAMA, I. Step-wise delignification response in an industrial two-stage oxygen-alkali delignification process. Pulp and Paper Canada, Toronto, v. 102, n. 6, p. 53-55. 2001.

ARGYROPOULOS, D. S., LIU, Y. The role and fate of lignin's condensed structures during oxygen delignification. Journal of Pulp and Paper Science, Montreal, v. 26, n. 3, p. 107113. 2000.BASSA, A. G. M. C. et al. Mixed brazilian Eucalyptus and Pinus species - bleaching evaluation. In: TAPPI Engineering, Pulping and Environment Conference, p. 1-14, PortlandOregon, August 24-27, 2008.

BERRY, R. Section IV: The technology of chemical pulp bleaching. Chapter 4: (Oxidative) Alkaline extraction. In. DENCE, C.W. and REEVE, D.W. Pulp bleaching - Principles and Practice. Tappi Press. Atlanta, Georgia. p. 291-320, 1996.

BOUCHARD, J. et al. Improved deresination during oxygen delignification. Pulp and Paper Canada, Toronto, v. 104, n. 2, p. 39-43. 2003.

BOUCHARD, J., WANG, J., BERRY, R. MgSO4 vs. $\mathrm{Mg}(\mathrm{OH}) 2$ as a cellulose protector in oxygen delignification. Holzforschung, Berlim, v. 65, n. 3, p. 295-301. 2011.

BRASILEIRO, L. B., COLODETTE, J. L., PIRÓ-VELOSO, D. A utilização de perácidos na deslignificação e no branqueamento de polpas celulósicas. Química Nova, São Paulo, v. 24, n. 6, p. 819-829. 2001.

CARTER, D. N. et al. Performance parameters of oxygen delignification. Tappi Journal, New York v. 80, n. 10, p. 111-117. 1997.

CHEN, S.-L., LUCIA, L.A. Improved method for evaluation of cellulose degradation. Journal of Wood Science, Tóquio, v. 49, n. 3, p. 285-288. 2003.

DE SOUZA, I. J. et al. Carbohydrates in oxygen delignification. Part I: Changes in cellulose crystallinity. Journal of Pulp and Paper Science, Montreal, v. 28, n. 5, p. 167-170. 2002.

FREDDO, A. et al. Elementos minerais em madeiras de Eucaliptos e Acácia negra e sua influência na indústria de celulose kraft branqueada. Ciência Florestal, Santa Maria, v. 9, n. 1, p. 193-209, 1999. GELLERSTEDT, G.; LINDFORS, E. L. Hydrophilic groups in lignin after oxygen bleaching. Tappi Journal, New York v. 70, n. 6, p. 119-122, 1987.

GEMZICKÁ, E. et al. Effect of $\mathrm{pH}$ and washing on 
calcium and magnesium distribution between pulp and filtrate. Chemical Papers, Bratislava, n. 64, v. 6, p.741-748, 2010.

GIERER, J. Formation and involvement of superoxide $\left(\mathrm{O}_{2}^{\cdot}-/ \mathrm{HO}_{2} \cdot\right)$ and hydroxyl $\left(\mathrm{OH}^{\cdot}\right)$ radicals in TCF bleaching processes: a review. Holzforschung, Berlim, v. 51, n. 1, p. 34-46. 1997. JIANG, Z.-H., VAN LIEROP, B., BERRY, R. Hexenuronic acid groups in pulping and bleaching chemistry. Tappi Journal, New York, v. 83, n. 1, p. 167-175. 2000.

JOHAKIMU, J. K. - Investigation of the potential to develop high pulp strength from high yield kraft pulp made from Pinus. 2007, 109 p. Thesis (Master Science in Engineering). School of Chemical Engineering of University of KwaZuluNatal and Forest Products Research. Centre of CSIR. Durban, South África.

LUCIA, L. A., RAGAUSKAS, A. J., CHAKAR, F. S. Comparative evaluation of oxygen delignification processes for low- and high-lignin-content softwood kraft pulps. Industrial \& Engineering Chemistry Research, Columbus, v. 41, n. 21, p. 5171-5180. 2002.

MCDONOUGH, T. J. Section IV: Chapter 1: The technology of chemical pulp bleaching. Oxygen delignification. In. DENCE, C. W.; REEVE, D. W. Pulp bleaching - Principles and Practice. Tappi Press. Atlanta, Georgia. p. 213-239. 1996.

MOE, S. T., RAGAUSKAS, A. J. Oxygen delignification of high-yield kraft pulp. Holzforschung, Berlim, v. 53, n. 4, p. 416-421. 1999.

POTUCEK, F.; PULCER, M. Displacement washing of pulp with urea solutions. Chemicals Papers, Bratislava, v. 60, n. 5, p.365-370. 2006.

RABELO, M. S. Tecnologias avançadas para pré-branqueamento de polpa kraft de eucalipto. 2006. 242f. Tese (Doutorado em Ciências Florestais) - Universidade Federal de Viçosa, Viçosa.

REEVE, D. W. Section I: Introduction. Chapter 1: Introduction to the principles and practice of pulp bleaching. In. DENCE, C. W. and REEVE, D. W.
Pulp bleaching - Principles and Practice. Tappi Press. Atlanta, Georgia. p. 3-24, 1996.

SAIBABA, K. et al. Technical upgradation in pulp washing \& bleaching stages to improve the final pulp quality and to reduce the pollution load. IPPTA Journal, Saharanpur, v. 21, n. 2, 2009.

SALOMÃO, K. G. Características e branqueabilidade de polpas kraft/polissulfeto de Eucalyptus e Pinus. 2001. 112f. Dissertação (Mestrado em Ciências Florestais) - Universidade Federal de Viçosa, Viçosa.

SILLANPÄÄ, M. Studies on washing in kraft pulp bleaching. 2005. Faculty of Technology, Department of Process and Environmental Engineering, University of Oulu, Finland.

SKOTNICOVÁ, I.; POTÙÈEK, F. Influence of wash liquid properties on the efficiency of pulp washing. Chemical Papers, Bratislava, v. 56, n. 6, p.369-373, 2002.

SUESS, H. U. Pulp bleaching today. Berlin: De Gruyter, 2010. 196p.

TAO. L. Oxygen delignification for southern softwood kraft pulps with high lignin. 2005. 162f. Theses (Master of seience in Chemical Engmeeing) - Chemical Engmeeing Department, Universiy of Maine, Maine.

TAPPI - Testing Procedures of Technical Association of the Pulp and Paper Industry. In: TAPPI Standard Method. Atlanta, USA. CD-ROM, 2001.

TORRES, L. F.; MELO, R.; COLODETTE, J. L. Bleached kraft pulp production from Pinus tecunumanii (Eguiluz e Perry). Revista Árvore, Viçosa, v. 29, n. 3, p. 489-494, 2005.

YOKOYAMA, T.; MATSUMOTO, Y.; MESHITSUKA, G., The role of peroxide species in carbohydrate degradation during oxygen bleaching. Part III: effect of metal ions on the reaction selectivity between lignin and carbohydrate model compounds. Journal of Pulp and Paper Science, Montreal, v. 25, n. 2, p. 42-46, 1999.

$\mathrm{ZOU}, \mathrm{H}$. et al. Influence of kraft pulping on the kinetics of oxygen delignification. Tappi Journal, New York, v. 83, n. 2, p. 65-71. 2000. 